\title{
Analytic Representation of Generalized Tempered Distributions by Wavelets
}

\author{
By \\ Byung Keun SoHN* and Dae Hyeon PAHK**
}

\begin{abstract}
The analytic representation of the generalized tempered distributions of exponential growth, $\mathcal{K}_{p}^{r \prime}$, is given in terms of series of analytic wavelets. These series converge uniformly on compact subsets of the upper and lower half planes.
\end{abstract}

\section{$\S 1$. Introduction}

The analytic representation of functions or distributions on the real line is usually given by a Cauchy type formula, but in some cases may also be given by an orthogonal series. This is evident for periodic functions and distributions for which trigonometric series may be used [5]. The more natural approch for arbitrary functions on the set of real numbers $R$ seems to be one involving wavelets. G. G. Walter has found an analytic representation of the tempered distributions of polynomial growth in terms of series of analytic wavelets [6].

In the past, the tempered distributions of polynomial growth were extended to various types of generalized tempered distributions of exponential growth [2], [3].

In this paper, we will find an analytic representation of the generalized tempered distributions of exponential growth in terms of series of analytic wavelets.

Communicated by T. Kawai, August 8, 1999. Revised May 18, 2000 and September 19, 2000 .

2000 Mathematics Subject Classification(s): 41A15, 46F20.

Key words and phrases: generalized tempered distribution, wavelet, analytic representation.

The first author was supported by Inje Research and Scholarship Foundation 98-99 and the second author was supported by KRF 99-005-D00009 and Yonsei Research Fund '97.

*Department of Mathematics, Inje University, Kyungnam, Kimhae, 621-749, (South) Korea.

** Department of Mathematics, Yonsei University, Seoul, 120-749, (South) Korea. 
These series converge uniformly on compact subsets of the upper and lower half planes.

\section{$\S 2$. The Generalized Tempered Distributions Space $\mathcal{K}_{p}^{\prime}(R)$}

We denote by $\mathcal{K}_{p}(R), p \geq 1$, the space of all functions $\phi \in C^{\infty}(R)$ such that

$$
\nu_{k}(\phi)=\sup _{x \in R, \alpha \leq k} e^{k|x|^{p}}\left|D^{\alpha} \phi(x)\right|<\infty, k=1,2, \ldots,
$$

where $D^{\alpha}=\frac{d^{\alpha}}{d x^{\alpha}}$. The topology in $\mathcal{K}_{p}(R)$ is defined by the family of the semi-norms $\nu_{k}$. Then $\mathcal{K}_{p}(R)$ becomes a Fréchet space and the embeddings $\mathcal{D} \hookrightarrow \mathcal{K}_{p} \hookrightarrow \mathcal{S} \hookrightarrow \mathcal{E}$ are continuous; here $\mathcal{E}$ denotes the space of all $C^{\infty}$ functions, $\mathcal{S}$ the space of the tempered ditsributions of polynomial growth and $\mathcal{D}$ the space of $C^{\infty}$-functions with compact supports. By $\mathcal{K}_{p}^{\prime}(R)$, we mean the space of continuous linear functionals on $\mathcal{K}_{p}(R)$. G. Sampson and Z. Zielezny characterized the distributions in $\mathcal{K}_{p}^{\prime}(R)$ by the growth at infinity [3]; a distribution $T \in \mathcal{D}^{\prime}$ is in $\mathcal{K}_{p}^{\prime}(R)$ if and only if there exist positive integers $\alpha, k_{0}$ and a bounded continuous function $f(x)$ on $R$ such that

$$
T=D^{\alpha}\left[e^{k_{0}|x|^{p}} f(x)\right]
$$

Definition 1. Let $r$ be a natural number and $p \geq 1$. We denote by $\mathcal{K}_{p}^{r}(R)$ the space of all functions $\phi \in C^{r}(R)$ such that

$$
\nu_{k}^{r}(\phi)=\sup _{x \in R, \alpha \leq r} e^{k|x|^{p}}\left|D^{\alpha} \phi(x)\right|<\infty, k=1,2,3, \ldots
$$

The topology of $\mathcal{K}_{p}^{r}(R)$ is defined by the family of semi-norms $\left\{\nu_{k}^{r}\right\}_{k=1,2, \ldots}$. By $\mathcal{K}_{p}^{r \prime}(R)$, we mean the space of continuous linear functionals on $\mathcal{K}_{p}^{r}(R)$. Each $S \in \mathcal{K}_{p}^{r \prime}(R)$ is characterized by

$$
S=D^{r}\left[e^{k_{0}|x|^{p}} f(x)\right]
$$

where $f(x)$ is a bounded continuous function on $R$ and $r, k_{0} \in N$, the set of natural numbers, by the same method of the above $\mathcal{K}_{p}^{\prime}$-case in [3, Theorem 2]. Similarly, we can define

$$
\mathcal{S}_{r}(R)=\left\{\theta(t) \in C^{r}(R) ;\left|D^{k} \theta(t)\right| \leq C_{p k}(1+|t|)^{-p}, p \in N, k=0,1, \ldots, r\right\}
$$

and its dual $\mathcal{S}_{r}^{\prime}(R)$. For further details, we refer to [3]. 


\section{$\S 3 . \quad$ Multiresolution Analysis of $L^{2}(R)$ Associated with $\phi \in \mathcal{K}_{p}^{r}(R)$}

Let $\phi \in \mathcal{K}_{p}^{r}(R)$. In order for it to qualify as a scaling function, there must be associated with $\phi$ a multiresolution analysis of $L^{2}(R)$, i.e., a nested sequence of closed subspaces $\left\{V_{m}\right\}_{m \in Z}$ for the set of integers $Z$ such that

(i) $\{\phi(t-n)\}$ is an orthonormal basis of $V_{0}$,

(ii) $\cdots \subset V_{-1} \subset V_{0} \subset V_{1} \subset \cdots \subset L^{2}(R)$,

(iii) $f \in V_{m} \Leftrightarrow f(2 \cdot) \in V_{m+1}$,

(iv) $\cap_{m} V_{m}=\{0\}, \overline{\cup_{m} V_{m}}=L^{2}(R)$.

Then $\phi$ has an expansion

$$
\phi(t)=\sum_{n} c_{n} \sqrt{2} \phi(2 t-n),\left\{c_{n}\right\} \in l^{2}, t \in R,
$$

where $l^{2}=\left\{\left\{c_{n}\right\} ; \sum_{n}\left|c_{n}\right|^{2}<\infty\right\}$. Once we have the scaling function $\phi \in$ $\mathcal{K}_{p}^{r}(R)$, we can obtain a mother wavelet $\psi$ such that $\{\psi(t-n)\}$ is an orthogonal basis of the space $W_{0}$, given by the orthogonal complement of $V_{0}$ in $V_{1}$. Also, $\psi$ has an expansion

$$
\psi(t)=\sum_{n} d_{n} \sqrt{2} \phi(2 t-n),\left\{d_{n}\right\} \in l^{2},
$$

for $d_{n}$ corresponding to $c_{n}$ in (3). We will adopt the construction of a mother wavelet defined by $d_{n}=(-1)^{n} \overline{c_{1-n}}$. If such a $\psi(t)$ can be found, then $\psi_{m n}(t)=$ $2^{\frac{m}{2}} \psi\left(2^{m} t-n\right)$ is an orthogonal basis of $W_{m}$ which is the orthogonal complement of $V_{m}$ in $V_{m+1}$.

Example. In [1], Corollary 5.5.3 states that it is impossible that $\psi$ has exponential decay and that $\psi \in C^{\infty}$, with all derivatives bounded, unless $\psi=0$. Hence there is no mother wavelet $\psi \in \mathcal{K}_{p}(R)$. So we will restrict our attention to $\mathcal{K}_{p}^{r}(R)$. Daubechies' compactly supported wavelets are examples of $\mathcal{K}_{p}^{r}(R)$, but Battle-Lemarié's wavelets (in the page 152 of [1]) are not $\mathcal{K}_{p}^{r}(R)$ wavelets even if they have exponential decay and smoothness.

The reproducing kernel of $V_{0}$ is given by

$$
q(x, t)=\sum_{n} \overline{\phi(x-n)} \phi(t-n),
$$

where $\phi(x)$ is the scaling function. The series and its derivatives with respect to $t$ of order $\leq r$ converge uniformly for $x \in R$ by the regularity of $\phi \in \mathcal{K}_{p}^{r}(R)$, i.e.,

$$
\left|\phi^{(\alpha)}(x)\right| \leq C_{\alpha k} e^{-k|x|^{p}}, \alpha=0,1, \ldots, r ; k=1,2, \ldots
$$


The reproducing kernel for $V_{m}$ is given by

$$
q_{m}(x, t)=2^{m} q\left(2^{m} x, 2^{m} t\right)
$$

Similarly, we can define the reproducing kernel $r_{m}(x, t)$ for $W_{m}$ by

$$
r_{m}(x, t)=2^{m} \sum_{n} \overline{\psi\left(2^{m} x-n\right)} \psi\left(2^{m} t-n\right),
$$

where $\psi(t)$ is the mother wavelet.

The sequence $\left\{q_{m}(x, t)\right\}$ is a delta sequence in $\mathcal{S}_{r}^{\prime}(R) \subset \mathcal{K}_{p}^{r^{\prime}}(R)$, i.e., $q_{m}(x, t) \rightarrow \delta(x-t)$. This follows from the fact that

$$
\int_{-\infty}^{\infty} q_{m}(x, t) \theta(t) d t \rightarrow \theta(x) \text { as } m \rightarrow \infty,
$$

for each $\theta \in \mathcal{K}_{p}^{r}(R) \subset \mathcal{S}_{r}(R)$, where the convergence is in the $L^{2}$-sense. These kernels have a number of interesting properties, some of which come out of the wavelet moment theorem. Since $\mathcal{K}_{p}^{r}(R) \subset \mathcal{S}_{r}(R)$, we have by [1],

Lemma 2. Let $\psi \in \mathcal{K}_{p}^{r}(R)$ with $\psi_{m n}(x)=2^{\frac{m}{2}} \psi\left(2^{m} x-n\right)$ an orthogonal system in $L^{2}(R)$. Then

$$
\int_{-\infty}^{\infty} x^{k} \psi(x) d x=0, \quad k=0,1, \ldots, r .
$$

Definition 3. We define the spaces $T_{0}$ and $U_{0}$ by $T_{0}=\{f ; f(t)=$ $\sum_{n} a_{n} \phi(t-n)$ for some sequence of complex numbers with $a_{n}=\mathcal{O}\left(e^{k_{1}|n|^{p}}\right)$ for some $\left.k_{1} \in N\right\}$ and $U_{0}=\left\{g ; g(t)=\sum_{n} a_{n} \psi(t-n)\right.$ for some sequence of complex numbers with $a_{n}=\mathcal{O}\left(e^{k_{1}|n|^{p}}\right)$ for some $\left.k_{1} \in N\right\}$. We denote by $T_{m}$ and $U_{m}$ their corresponding dilation spaces, i. e., $f \in T_{0} \Leftrightarrow f\left(2^{m} t\right) \in T_{m}$ and $g \in U_{0} \Leftrightarrow g\left(2^{m} t\right) \in U_{m}$.

We may expect that a multiresolution analysis of $\mathcal{K}_{p}^{r \prime}(R)$ exists, namely,

$$
\cdots \subset T_{-m} \cdots \subset T_{-1} \subset T_{0} \subset T_{1} \cdots \subset T_{m} \subset \cdots \subset \mathcal{K}_{p}^{r \prime}(R)
$$

and

$$
\overline{\cup_{m} T_{m}}=\mathcal{K}_{p}^{r^{\prime}}(R),
$$

where the closure is in the topology of $\mathcal{K}_{p}^{r \prime}(R)$.

Now in [4], we have found the expansion in orthogonal wavelets from $L^{2}(R)$ to $\mathcal{K}_{p}^{r \prime}(R)$. 
Theorem 4. Let the scaling function $\phi \in \mathcal{K}_{p}^{r}(R)$ satisfy the dilation equation (3) with $c_{k}=\mathcal{O}\left(e^{-l|k|^{p}}\right)$ for all $l \in N$, and have an associated multiresolution analysis in $L^{2}(R)$; let $\psi \in \mathcal{K}_{p}^{r}(R)$ be the mother wavelet given in (4). Then there exists a multiresolution analysis (6) of closed dilation subspaces $\left\{T_{m}\right\}$ whose union is dense in $\mathcal{K}_{p}^{r \prime}(R)$; the closed subspace $U_{m}$ in Definition 3 is a complementary subspace of $T_{m}$ in $T_{m+1}$ and

$$
T_{m}=U_{0} \oplus U_{1} \oplus \cdots \oplus U_{m} \oplus T_{0},
$$

where $\oplus$ denotes the nonorthogonal direct sum.

\section{$\S 4$. Analytic Representation of Distributions of $\mathcal{K}_{p}^{r \prime}$ by Wavelets}

A quasi-positive delta sequence is a sequence $\left\{\delta_{m}(\cdot, y)\right\}$ of functions in $L^{1}(R)$ with a parameter $y \in R$ which satisfies the following:

(a) there is a $C>0$ such that

$$
\int_{-\infty}^{\infty}\left|\delta_{m}(x, y)\right| d x \leq C, y \in R, \quad m \in N
$$

(b) there is a $c>0$ such that

$$
\int_{y-c}^{y+c} \delta_{m}(x, y) d x \rightarrow 1
$$

uniformly on compact subsets of $R$, as $m \rightarrow \infty$;

(c) for each $\gamma>0$,

$$
\sup _{|x-y| \leq \gamma}\left|\delta_{m}(x, y)\right| \rightarrow 0 \quad \text { as } \quad m \rightarrow \infty .
$$

Then since $\mathcal{K}_{p}^{r}(R) \subset \mathcal{S}_{r}(R)$, we have the following important lemmas as in [7]:

Lemma 5. Let $\left\{\delta_{m}(x, y)\right\}$ be a quasi-positive delta sequence and let $f \in$ $L^{1}(R)$ be continuous on $(a, b)$. Then

$$
f_{m}(y)=\int_{-\infty}^{\infty} \delta_{m}(x, y) f(x) d x \rightarrow f(y) \quad \text { as } \quad m \rightarrow \infty
$$

uniformly on compact subsets of $(a, b)$.

Lemma 6. If the scaling function $\phi \in \mathcal{K}_{p}^{r}(R)$, then the reproducing kernel $q_{m}(x, y)$ and $K_{m}(x, t)=\frac{(x-t)}{\alpha !} \frac{\partial^{\alpha}}{\partial t^{\alpha}} q_{m}(x, t)$ for $\alpha \in N, 0 \leq \alpha \leq r$, are quasi-positive delta sequences on $R$. 
In order to represent an element of $\mathcal{K}_{p}^{r \prime}(R)$ by series of analytic wavelets, we impose conditions on the scaling function $\phi$ again. Since $\mathcal{K}_{p}^{r}(R) \subset L^{2}(R)$, an analytic represetation of $\phi$ is given by

$$
\phi^{ \pm}(z)=\frac{1}{2 \pi i} \int_{-\infty}^{\infty} \frac{\phi(x)}{x-z} d x, \operatorname{Im} z \gtrless 0,
$$

where $\phi^{ \pm}$are analytic in the upper half-plane and the lower half-plane, respectively. An analytic representation of the mother wavelet is also given by

$$
\psi^{ \pm}(z)=\frac{1}{2 \pi i} \int_{-\infty}^{\infty} \frac{\psi(x)}{x-z} d x, \operatorname{Im} z \gtrless 0,
$$

and the analytic wavelets $\psi_{m n}^{ \pm}$are obtained by dilation and translation of $\psi^{ \pm}$. Now, we define $T_{0}^{ \pm}=\left\{f(z)=\sum_{n} a_{n} \phi^{ \pm}(z-n) ; a_{n}=\mathcal{O}\left(e^{l_{0}|n|^{p}}\right)\right.$ for some $l_{0} \in$ $N\}$ and we denote by the subspaces $T_{m}^{ \pm}$of $T_{0}^{ \pm}$the corresponding dilation spaces. Then the spaces $T_{m}^{+}$and $T_{m}^{-}$are composed of analytic functions in the upper and the lower half-planes, respectively, whose boundary functions are continuous functions of exponential growth. Since $\overline{\cup T_{m}}=\mathcal{K}_{p}^{r \prime}(R)$, we might expect to obtain an analytic representation of $f \in \mathcal{K}_{p}^{r \prime}(R)$ in terms of wavelets,

$$
f^{+}(z)=\sum_{n=-\infty}^{\infty} a_{n} \phi^{+}(z-n)+\sum_{m=0}^{\infty} \sum_{n=-\infty}^{\infty} b_{m n} 2^{\frac{m}{2}} \psi^{+}\left(2^{m} z-n\right),
$$

where the first series may not converge. Since an analytic representation is a continuous map from $\mathcal{K}_{p}^{\text {r'}}(R)$ to a corresponding space of analytic functions and $f_{m}(x)=\left(f, q_{m}(x, t)\right) \rightarrow f(x)=D^{r} F(x)$ in $\mathcal{K}_{p}^{r \prime}(R)$ for a continuous function of exponential growth $F(x)$ [cf. (2)] by Lemmas 5 and $6, f_{m}^{+}(z) \rightarrow f^{+}(z)$ uniformly on bounded subsets of the upper half-plane. Moreover, $f^{+}(z)=D_{z}^{r} F^{+}(z)$, where $F^{+}(z)$ is an analytic representation of $F(z)$, and is given by

$$
F^{+}(z)=\frac{1}{2 \pi i} \int_{-\infty}^{\infty} \frac{F(x)}{x-z} e^{-k|x|^{p}} e^{k|z|^{p}} d x,
$$

for a sufficiently large $k$ such that $F(x) e^{-k|x|^{p}} \in L^{2}(R)$.

We may express $f_{m}$ as

$$
f_{m}=f_{0}+f_{m}-f_{0}=f_{0}+\sum_{k=0}^{m-1} \sum_{n=-\infty}^{\infty} b_{k n} \psi_{k n},
$$

and if the inner sum converges,

$$
f_{m}^{+}(z)-f_{0}^{+}(z)=\sum_{k=0}^{m-1} \sum_{n=-\infty}^{\infty} b_{k n} \psi_{k n}^{+}(z)+g_{m}(z),
$$

where $g_{m}(z)$ is an entire function. 
Lemma 7. Let $\psi \in \mathcal{K}_{p}^{r}(R)$ and $b_{n}=\mathcal{O}\left(e^{k|n|^{p-\epsilon}}\right)$ for any $k \in N$ and some $\epsilon>0$. Then

$$
\sum_{n=-\infty}^{\infty} b_{n} \psi^{+}(z-n)
$$

converges uniformly on compact subsets of the upper half-plane.

Proof. The proof is based on the moment property, Lemma 2,

$$
\int_{-\infty}^{\infty} x^{l} \psi(x) d x=0, l=0,1, \ldots, r .
$$

Hence, for any $k \in N$ and a natural number $p \leq r+1$,

$$
\begin{aligned}
e^{k|z|^{p}} & \psi^{+}(z) \\
= & \frac{1}{2 \pi i} \int_{-\infty}^{\infty} \frac{e^{k|z|^{p}}}{z^{p}} \cdot \frac{z^{p}}{x-z} \psi(x) d x \\
= & \frac{1}{2 \pi i} \int_{-\infty}^{\infty} \frac{e^{k|z|^{p}}}{z^{p}} \cdot \frac{z^{p}-x^{p}}{x-z} \psi(x) d x \\
& \quad+\frac{1}{2 \pi i} \int_{-\infty}^{\infty} \frac{e^{k|z|^{p}}}{z^{p}} \cdot \frac{x^{p}}{x-z} \psi(x) d x \\
= & -\frac{1}{2 \pi i} \int_{-\infty}^{\infty} \frac{e^{k|z|^{p}}}{z^{p}} \cdot\left(x^{p-1}+z x^{p-2}+\cdots+z^{p-2} x+z^{p-1}\right) \psi(x) d x \\
= & \quad+\frac{1}{2 \pi i} \int_{-\infty}^{\infty} \frac{e^{k|z|^{p}}}{z^{p}} \cdot \frac{x^{p}}{x-z} \psi(x) d x \\
\hline & \frac{e^{k|z|^{p}}}{z^{p}} \cdot \frac{x^{p}}{x-z} \psi(x) d x
\end{aligned}
$$

holds. By the growth condition of $\psi \in \mathcal{K}_{p}^{r}(R), \quad\left|e^{k|z|^{p}} \psi^{+}(z)\right|$ is uniformly bounded on compact subsets of the half-plane $\operatorname{Imz} \geq \epsilon>0$ for any $k \in N$ and a natural number $p \leq r+1$. Hence, the preceding fact holds for any $k \in N$ and any $p \leq r+1$. Thus the conclusion follows.

Theorem 8. Let $f \in \mathcal{K}_{p}^{s^{\prime}}(R), \phi, \psi \in \mathcal{K}_{p}^{r}(R), s<r$ and let $b_{m n}=$ $\left\langle f, \psi_{m n}\right\rangle, m=0,1,2, \ldots ; n=0, \pm 1, \pm 2, \ldots$ be the wavelet coefficients of $f$. Then an analytic representation of $f$ is given by

$$
f^{+}(z)=f_{0}^{+}(z)+\sum_{m=0}^{\infty} \sum_{n=-\infty}^{\infty} b_{m n} \psi_{m n}^{+}(z)
$$

where the series converges uniformly on compact subsets of the half-plane Im $z$ $\geq 1$ and $f_{0}^{+}(z)$ is an analytic representation of $f_{0}$, the projection of $f$ on $T_{0}$. 
Proof. First, we will estimate $\left|b_{m n}\right|$. Each $f \in \mathcal{K}_{p}^{s^{\prime}}(R)$ is characterized by

$$
f=D^{s}\left[e^{k_{0}|x|^{p}} \mu\right]
$$

for some integer $k_{0}$ and finite measure $\mu$ on $R$. Each $\psi \in \mathcal{K}_{p}^{r}(R)$ satisfies

$$
\left|\psi^{(l)}(x)\right| \leq C_{j} e^{-j|x|^{p}}, l=1,2, \ldots, r ; j \geq 0 .
$$

If we use integration by parts $s$-times and the inequality $(a+b)^{p} \leq 2^{p}\left(a^{p}+\right.$ $\left.b^{p}\right), a, b>0$, we have, for $m>1$,

$$
\begin{aligned}
\left|b_{m n}\right| & \leq \int_{-\infty}^{\infty}\left|D^{s}\left[e^{k_{0}|x|^{p}}\right] \psi_{m n}(x)\right| d|\mu| \leq \int_{-\infty}^{\infty} e^{k_{0}|x|^{p}}\left|\psi_{m n}^{(s)}(x)\right| d|\mu| \\
& \leq \int_{-\infty}^{\infty} e^{k_{0}|x|^{p}} c_{k_{0}} 2^{\frac{m}{2}+s m} e^{-k_{0}\left|2^{m} x-n\right|^{p}} d|\mu| \\
& \leq \int_{-\infty}^{\infty} e^{2^{p} k_{0}\left|x-n 2^{-m}\right|^{p}} e^{2^{p} k_{0}\left|n 2^{-m}\right|^{p}} c_{k_{0}} 2^{\frac{m}{2}+s m} e^{-k_{0}\left(2^{m}\right)^{p}\left|x-n 2^{-m}\right|^{p}} d|\mu| \\
& \leq c_{k_{0}}^{\prime} 2^{\frac{m}{2}+s m} e^{2^{p} k_{0}\left|n 2^{-m}\right|^{p}} .
\end{aligned}
$$

By the fact in the proof of Lemma 7, on every compact subset $K$ of the half-plane $\operatorname{Im} z \geq 1$, there exists a constant $c$ such as $\left|\psi^{+}(z)\right| \leq c e^{-k|z|^{p}}$ for any $k \in N$. Let $k>k_{0}$ and $M=\max \left(7, s+\frac{3}{2}\right)$. Then we have, for every $z \in K$,

$$
\begin{aligned}
& \sum_{m=0}^{\infty} \sum_{n=-\infty}^{\infty}\left|b_{m n} \psi_{m n}^{+}(z)\right| \\
& \quad \leq \sum_{m=0}^{\infty} \sum_{n=-\infty}^{\infty} c_{k_{0}}^{\prime} e^{\left(\frac{1}{2}+s\right) m} e^{2^{p} k_{0}\left|n 2^{-m}\right|^{p}} c 2^{\frac{m}{2}} e^{-k\left|2^{m} z-n\right|^{p}} \\
& \leq \sum_{m=0}^{\infty} \sum_{n=-\infty}^{\infty} c_{k_{0}}^{\prime} e^{\left(\frac{3}{2}+s\right) m} e^{2^{p} k_{0}\left|n 2^{-m}\right|^{p}} c e^{-k\left(2^{m}\right)^{p}\left|\left(n 2^{-m}-R e z\right)^{2}+1\right|^{\frac{p}{2}}} 2^{-\frac{m}{2}} \\
& \leq\left\{\sum_{m=0}^{M} \sum_{n=-\infty}^{\infty}+\sum_{m=M+1}^{\infty} \sum_{n=-\infty}^{\infty}\right\} c c_{k_{0}}^{\prime} e^{\left(\frac{3}{2}+s\right) m} e^{2^{p} k_{0}\left|n 2^{-m}\right|^{p}} \\
& \quad \times e^{-k 2^{p}\left|\left(n 2^{-m}-R e z\right)^{2}+1\right|^{\frac{p}{2}}} e^{-k m^{2 p}\left|\left(n 2^{-m}-R e z\right)^{2}+1\right|^{\frac{p}{2}}} 2^{-\frac{m}{2}} \\
& \leq \sum_{m=M+1}^{\infty} C_{k_{0}, z^{-\frac{m}{2}}}^{\infty}<\infty
\end{aligned}
$$

where we use $\left(2^{m}\right)^{p} \geq 2^{p}+\left(2^{m-1}\right)^{p} \geq 2^{p}+m^{2^{p}}$ for $m \geq 7$. Hence the series $\sum_{m=0}^{\infty} \sum_{n=-\infty}^{\infty} b_{m n} \psi_{m n}^{+}(z)$ converges uniformly on compact subsets of the halfplane $\operatorname{Im} z \geq 1$.

Now, by taking the limit in (7) as $m \rightarrow \infty$, we have

$$
f^{+}(z)=f_{0}^{+}(z)+\sum_{k=0}^{\infty} \sum_{n=-\infty}^{\infty} b_{k n} \psi_{k n}^{+}(z)+g_{\infty}(z)
$$

where $g_{\infty}(z)=\lim _{m \rightarrow \infty} g_{m}(z)$ is an entire function. Since an analytic representation plus an entire function is an analytic representation, we can drop $g_{\infty}$ in $(7)$. 
Remark. We have only worked out the convergence for $f^{+}$but proof for $f^{-}$is parallel. Then by the same method as in the proof of Theorem 8, an analytic representation of $f$ is given by

$$
f^{-}(z)=f_{0}^{-}(z)+\sum_{m=0}^{\infty} \sum_{n=-\infty}^{\infty} b_{m n} \psi_{m n}^{-}(z)
$$

where the series converges uniformly on compact subsets of the half-plane Im $z \leq-1$ and $f_{0}^{-}(z)$ is an analytic representation of $f_{0}$, the projection of $f$ on $T_{0}$.

\section{Acknowlegement}

The authors thank the referee for careful reading and valuable suggestions for the revision of this paper.

\section{References}

[1] Daubechies, I., Ten Lectures on Wavelets, SIAM, CBMS 61, Philadelphia, 1992.

[2] Hasumi, M., Note on the n-dimensional tempered ultra-distributions, Tohoku Math. J., 13 (1961), 94-104.

[3] Sampson, G. and Zielezny, Z., Hypoelliptic convolution equations in $\mathcal{K}_{p}^{\prime}$, Trans. A. M. S., 223 (1976), 133-154.

[4] Sohn, Byung Keun and Pahk, Dae Hyeon, Wavelets in the generalized tempered distributions, Tsukuba J. Math., 23 (1999), 529-538.

[5] Walter, G. G., Fourier series and analytic representation of distributions, SIAM Rev., 12 (1970), 272-276.

[6] - Analytic representations with wavelet expansions, Complex Variables Theory Appl., 26 (1994), 235-243.

[7] - Pointwise convergence of wavelet expansions, J. Approx. Theory, 80 (1995), $108-118$. 